\title{
POTENCIAL ENERGÉTICO DA BIOMASSA DE CANA-DE-AÇÚCAR EM UMA USINA SUCROENERGÉTICA
}

\author{
Lucas Amaro dos SANTOS ${ }^{1}$ \\ José Edmundo Accioly de SOUZA ${ }^{2}$ \\ Aline da Silva Ramos BARBOZA ${ }^{3}$ \\ Vinicius Santos Gomes da SILVA ${ }^{4}$ \\ Paulo Ricardo Aprígio CLEMENTE ${ }^{5}$
}

\begin{abstract}
${ }^{1}$ EngenheiroQuímico, departamento de Engenharia Química,Universidade Federal de Alagoas - UFAL - Maceió/AL, e-mail: lucas_smc@hotmail.com.

${ }^{2}$ Engenheiro Químico, doutor pelo Programa de Pós-Graduação em Química da Universidade Federal de Alagoas UFAL - Maceió/AL, e-mail: edmundoaccioly@msn.com.

${ }^{3}$ Engenheira Civil, doutora em Engenharia Civil (Engenharia de Estruturas) pela Escola de Engenharia de São Carlos EESC/USP - São Paulo/SP, email: aline@lccv.ufal.br

${ }^{4}$ Engenheiro Agrônomo, doutorando pelo Programa de Pós-Graduação em Ciência do Solo da Universidade Federal Rural de Pernambuco - UFRPE - Recife/PE, e-mail: vinicius.agro2008.1@ gmail.com.

${ }^{5}$ Engenheiro Agrônomo, doutorando pelo Programa de Pós-Graduação em Produção Vegetal da Universidade Federal de Alagoas - UFAL - Maceió/AL, e-mail: pauloclemente_645@ hotmail.com.
\end{abstract}

Recebido em: 07/06/2016 - Aprovado em: 05/11/2016 - Disponibilizado em: 18/12/2016

\begin{abstract}
RESUMO:
Objetivou-se com o presente estudo avaliar o potencial energético de pontas, folhas e bagaço de cana em uma usina sucroenergética na Zona da Mata de Alagoas. Para realização do estudo, foram levantadas informações sobre a quantidade de resíduos de cana produzidos em AL e na Usina Sinimbu, posteriormente realizou-se a amostragem de 20 $\mathrm{kg}$ de cana em canavial localizado no município de Campo Alegre/AL para avaliação física, química e energética dos resíduos da biomassa da parte aérea das plantas, com bases nos resultados determinou-se o balanço mássico e energético para a Usina. Os resíduos de biomassa apresentam alto potencial energético, constituindo assim em alternativa econômica para o setor frente ao cenário de crise econômica verificado em grande parte das unidades do setor sucroenergético.
\end{abstract}

Palavras -chave: geração de energia, economia,balanço mássico.

\section{POTENTIAL ENERGY OF CANE SUGAR IN A BIOMASS PLANT SUGARCANE}

\begin{abstract}
:
The objective of the present study was to evaluate the energy potential of tips, leaves and sugar cane bagasse in a sugar and ethanol plant in Alagoas Forest Zone. For the study, information was raised about the amount of sugarcane waste produced in AL and Plant Sinimbu subsequently held sampling 20kg cane in cane field located in the municipality of Campo Alegre / AL for physical evaluation, and chemical energy from waste biomass of the shoot, with bases in the results determined the mass balance and energy to the plant. The biomass residues have high energy potential , thus constituting economic alternative to the front sector to the economic crisis scenario found in most units of the sugarcane industry.
\end{abstract}

Keywords: power generation, economy, mass balance. 


\section{Introdução}

Atualmente o setor sucroenergético no Nordeste brasileiro enfrenta uma crise agrícola (redução na produtividade) e industrial (fechamento de usinas). Assim, alternativas que possibilitem aumentar a lucratividade na canavicultura e torná-la mais competitiva são necessárias. A utilização de resíduos da biomassa para geração de energia constitui-se em uma possibilidade de exploração econômica para o setor canavieiro, uma vez que pontas, folhas, e bagaço de cana tem alto potencial energético.

A energia de biomassa constitui-se principalmente de substâncias de origem orgânica (vegetal, animal e microrganismos), sendo uma energia limpa e renovável. Em Alagoas têm-se diversas fontes, sendo as provenientes de resíduos agrícolas, de resíduos florestais, entre outros. A cana-de-açúcar gera resíduos agrícolas (palhas) e também resíduos agroindustriais (bagaço) que já são aproveitados nas caldeiras das usinas e destilarias para fins energéticos.

A utilização de resíduos para geração de energia já é uma realidade, pois além de ter um grande potencial, ajuda o meio ambiente. Neste quesito, o setor sucroenergético pode contribuir muito para a necessidade energética do país. Em Alagoas, o setor é o maior contribuinte para o PIB do estado. Todas as usinas do estado são autossuficientes em energia e algumas delas ainda exportam para rede distribuidora.
Embora o setor sucroenergético demonstre um grande potencial de cogeração de energia, apresentando atrativos econômicos e ambientais, devido à utilização do bagaço de cana como combustível, a produção de energia elétrica para a venda às concessionárias ainda é incipiente, além disso poucos trabalhos na literatura discorrem sobre a capacidade dos resíduos da biomassa de cana na produção energética no nordeste, desse modo estudos que avaliem a potencialidade de resíduos de biomassa em usinas sucroenergéticas são importantes para contribuir em uma expansão da utilização desses materiais vegetais para as usinas, constituindo assim em alternativa econômica para as usinas frente ao cenário de crise econômica e energética ocorrida recentemente.

Ante ao exposto, objetivou-se com este trabalho avaliar o potencial energético dos resíduos da biomassa de cana-de-açúcar em uma usina sucroenergética deAlagoas, por meio da quantificação da quantificação da energia que pode ser gerada a partir de bagaço, pontas e folhas.

\section{Materiais e Métodos}

Para a realização da pesquisa, foram levantadas informações sobre a quantidade de biomassa da parte aérea de cana-de-açúcar produzidos em Alagoas (CONAB, 2014), a partir destes dados realizou-se uma estimativa da quantidade média de resíduos da 
canavicultura (tabela 1) gerados no estado de Alagoas, segundo a metodologia de Souza (2011).

Tabela 1: Quantidade de resíduos de cana-deaçúcar em Alagoas safra 14/15.

\begin{tabular}{ccc}
\hline Resíduo & $\begin{array}{c}\text { Produtividade } \\
(\mathrm{t}) / \text { ha }\end{array}$ & $\begin{array}{c}\text { Quantidade total } \\
\text { gerada na safra }\end{array}$ \\
\hline $\begin{array}{c}\text { Palhas e } \\
\text { Pontas }\end{array}$ & 11,64 & $4.484 .892,00$ \\
Bagaço & 14,55 & $5.606 .115,00$ \\
\hline
\end{tabular}

Após o levantamento, foram coletadas amostrasde aproximadamente $20 \mathrm{~kg}$ da biomassa da parte aérea de cana-de-açúcar em canavial pertencente a usina Sinimbú na cidade de Campo Alegre - AL, o material vegetal amostrado, foi identificado e levado para Universidade Federal de Alagoas para realização de análises físico-químicas no laboratório de biocombustíveis e energia (LABEN). No laboratório determinou-se a umidade e o poder calorífero. $\mathrm{O}$ poder calorífico superior das amostras foi determinado seguindo as normas ABNT NBR 8633/84, utilizando-se um Calorímetro IKA C 200 (ASTM D-2382). A Figura 2 mostra o Calorímetro IKA C 200 instalado no Laboratório de Biocombustíveis e Energia (LABEN) da Universidade Federal de Alagoas (UFAL).

$\mathrm{Na}$ determinação do PCS, todas as análises foram realizadas em triplicata, adotando um tempo de queima aproximadamente 08 (oito) minutos. Após o término do processo de queima. A partir do valor do PCS obtido no calorímetro, calculouse o PCI utilizando-se da relação matemática entre o PCS e o PCI, dada pela Equação II:Onde:PCI - Poder Calorífico Inferior $[\mathrm{kJ} / \mathrm{kg}]$ em base seca;PCS - Poder Calorífico Superior $[\mathrm{kJ} / \mathrm{kg}]$, determinado com o uso do Calorímetro;H - Teor de hidrogênio do combustível [kg/kg] em base seca; $\mathrm{U}$ - Teor de umidade do combustível $\left[\mathrm{kg} \quad\left(\mathrm{H}_{2} \mathrm{O}\right) / \mathrm{kg}\right.$ (combustível seco), determinado através da estufa de secagem.Usou-se para a variável $H$ o valor de 6,5 (RANADE ; KAWRE, 2010).

$$
P C I=P C S-2440(9 H+U)
$$

O teor de umidade foi determinado seguindo as Normas ABNT NBR 8112. Foi utilizado um cadinho de porcelana de massa conhecida, no qual foram colocados 01 (um) g da amostra. Após, o sistema foi colocado em uma estufa de secagem durante 90 minutos a $105^{\circ} \mathrm{C}$. Passado o tempo programado, deixouse a amostra esfriar no dessecador para que a mesma ficasse com peso constante.A massa que foi perdida na secagem corresponde a massa de água. Desta forma foi calculado o teor de umidade em porcentagem.

A partir do levantamento teórico e dos resultados das analise físico - químicas realizou-se estimativas da quantidade de energia térmica teórica, pela equação.

$$
Q=\Delta H(I I I)
$$


Para calcular a quantidade teórica de vapor que pode ser produzida nestas caldeiras, foi preciso realizar uma série de considerações. 1) Combustão completa do bagaço; 2) toda energia oriunda da queima seja transferida para água; 3) não haja perda de calor para fora do sistema; 4) Alimentação constante de bagaço e água; 5) não haja purga no sistema; 6) Estado estacionário.

Fazendo estas considerações podemos utilizar a equação abaixo para calcular a quantidade de energia que pode ser gerada utilizando a alimentação que é usada nas caldeiras.

$$
\dot{Q}=\dot{m} x P C I(\mathrm{IV})
$$

Onde: $\dot{Q}$ : Quantidade de energia por unidade de tempom: Alimentação de combustível por unidade de tempoPCI: Poder calorifico inferior em $\mathrm{KJ} / \mathrm{Kg}$

Para encontrar a eficiência da caldeira utilizamos um balanço energético baseado na equação III para calcular a quantidade de vapor teórico através da equação $\mathrm{V}$ abaixo.

$$
\begin{array}{r}
\dot{Q}=\dot{m} C p 1(\text { Tsat }-T i)+\dot{m} \times L v \\
+\dot{m} C p 2(T f-T s a t)
\end{array}
$$

Onde: $\dot{Q}$ : Quantidade de energia por unidade de tempo $(\mathrm{KJ} / \mathrm{h}) \dot{m}$ : vazão mássica $(\mathrm{Kg} / \mathrm{h}) \mathrm{Cp} 1$ : Calor específico da água $(\mathrm{KJ} / \mathrm{Kg}$ $\left.{ }^{\circ} \mathrm{C}\right)$ Lv: Calor específico de vaporização (KJ/Kg) Cp2: Calor específico do vapor superaquecido $\left(\mathrm{KJ} / \mathrm{Kg}{ }^{\circ} \mathrm{C}\right) \mathrm{Ti}$ : Temperatura de entrada $\left({ }^{\circ} \mathrm{C}\right)$ Tsat: Temperatura de saturação $\left({ }^{\circ} \mathrm{C}\right)$ Tf: Temperatura de saída $\left({ }^{\circ} \mathrm{C}\right)$
Calculou-se a eficiência de geração da caldeira através da fórmula a seguir.Onde: $\varepsilon \%$ : Eficiência em porcentagem; R: Vazão de vapor real da caldeira; T: Vazão de vapor teórico.

$$
\varepsilon \%=(R \div T) \times 100(\mathrm{VI})
$$

\section{Resultados e Discussão}

A umidade inicial das palhas e pontas analisadas foi de $77 \%$ e para o bagaço 86\%.Com as análises laboratoriais foi possível se fazer uma estimativa da quantidade de energia que pode ser gerada a partir destes resíduos. Sabendo-se que o PCS (Poder Calorífico Superior) das palhas e pontas analisadas com umidade de $14,82 \%$ é de $14.941 \mathrm{KJ} /$, pode-se utilizar a equação II e de modo empírico calcular o valor do PCI. Sendo assim obteve-se o valor de 13.151,99 $\mathrm{KJ} / \mathrm{Kg}$.De forma análoga, foi realizado o cálculo para o bagaço, cujo PCS com umidade de $50 \%$ é de $9.532,29 \mathrm{KJ} / \mathrm{Kg}$ e PCI $6.884,89$ $\mathrm{KJ} / \mathrm{Kg}$.

Através destes resultados podemos notar que as palhas e pontas nestes parâmetros de umidade superam o bagaço em capacidade energética em pouco mais de $52 \%$. Com os dados do PCI das palhas e pontas e do PCI do bagaço, foi possível realizar uma estimava da quantidade de energia térmica que pode ser gerada no estado de Alagoas conforme a Tabela 2. 
Tabela 2: Características físico-químicas e quantidade de energia que pode ser gerada a partir dos resíduos na safra 2014/2015.

\begin{tabular}{cccccc}
\hline Resíduo & $\begin{array}{c}\text { Quantidade } \\
\text { (toneladas) }\end{array}$ & $\begin{array}{c}\text { PCS } \\
(\mathrm{KJ} / \mathrm{Kg})\end{array}$ & $\begin{array}{c}\text { Umidade } \\
(\%)\end{array}$ & $\begin{array}{c}\text { PCI } \\
(\mathrm{KJ} / \mathrm{Kg})\end{array}$ & $\begin{array}{c}\text { Energia } \\
(\mathrm{KJ})\end{array}$ \\
\hline $\begin{array}{c}\text { Palhas } \\
\text { e Pontas }\end{array}$ & $4.484 .892,00$ & $14.941,00$ & 14,82 & $13.151,99$ & $58.985 .254 .735 .080,00$ \\
Bagaço & $5.606 .115,00$ & $9.532,29$ & 50,00 & $6.884,89$ & $38.597 .485 .102 .350,00$ \\
TOTAL & $10.091 .010,00$ & & & & $97.582 .739 .837 .430,00$ \\
\hline
\end{tabular}

Tabela 3: Consumo de bagaço nas caldeiras e geração de vapor.

\begin{tabular}{|c|c|c|c|c|c|}
\hline Consumo & Consumo & Geração & Geração & Vapor para & Vapor para \\
\hline \multirow[t]{2}{*}{ Bagaço Total } & Bagaço por & Vapor Total & Vapor por & Indústria & Geração de \\
\hline & Caldeira & & Caldeira & & Energia \\
\hline & ------- & ---------- & /h------- & ---------- & --- \\
\hline 120 & 40 & 240 & 80 & 100 & 140 \\
\hline
\end{tabular}

Utilizando todos os dados e substituindo na equação IV, temos que a quantidade de energia que pode ser gerada por caldeira a partir desta quantidade de bagaço alimentado na umidade de $50 \%$ é de 275.395.600 KJ/h.

A partir dos dados de funcionamento das caldeiras da usina, sabe-se que a temperatura de entrada da água é de $110^{\circ} \mathrm{C}$ e a temperatura de saída do vapor superaquecido é de $359{ }^{\circ} \mathrm{C}$ e pressão de $21 \mathrm{bar}\left(\mathrm{Kgf} / \mathrm{cm}^{2}\right)$.

Com estes dados, e realizandoconsulta de tabelas de vapor, temos que:

$\mathrm{Cpl}=4,18 \mathrm{KJ} / \mathrm{Kg}{ }^{\circ} \mathrm{C}$;

$\mathrm{Cp} 2=2,23 \mathrm{KJ} / \mathrm{Kg}$

$L v=1883,63 \mathrm{KJ} / \mathrm{Kg} ;$ Tsat $=214,9^{\circ} \mathrm{C}$.
Substituindo estes valores na fórmula $\mathrm{V}$ e isolando $\dot{m}$, obtemos assim a quantidade teórica de vapor que se pode gerar nestas condições que é 104.180,17 Kg/h ou aproximadamente 104,18 toneladas por hora. Utilizando a equação VI, encontrou-se uma eficiência de geração de vapor de $76,79 \%$. A partir desta eficiência foi possível realizar uma estimativa da quantidade de vapor real que pode ser gerada com a palha, alimentando a mesma vazão que é alimentada o bagaço. Sendo assim, utilizando a equação IV, para palha a quantidade de energia é 526.079.680 $\mathrm{KJ} / \mathrm{h}$. A partir da equação $\mathrm{V}$ a vazão teórica de vapor é aproximadamente 199 toneladas por hora e por fim utilizando à equação VI a vazão real de vapor gerado na caldeira seria de 152,8 
toneladas de vapor por hora, que forneceria um aumento de geração de $91 \%$ em comparação com a geração a partir do bagaço.

Como dito anteriormente, a usina conta com 3 caldeiras, juntas elas produzem 240 toneladas de vapor por hora e um consumo de 120 toneladas de bagaço por hora. Sendo assim, utilizando palhas e pontas uma única caldeira produziria 152,8 toneladas de vapor por hora. Considerando as 3 caldeiras teríamos uma produção de 458,4 toneladas de vapor por hora. Portanto, para suprir a necessidade energética atual da usina, utilizando somente as palhas e pontas, bastaríamos entrar com uma alimentação de $62,82 \mathrm{t} / \mathrm{h}$ de palhas e pontas, ou seja, uma economia de 57,18 toneladas de combustível, o que equivale a $47,65 \%$.

Para geração de energia, há um consumo atual de $140 \mathrm{t} / \mathrm{h}$ de vapor para geração de $18 \mathrm{MW} / \mathrm{h}$, contando a geração dos 3 turbo geradores existentes. Com a utilização das palhas e pontas, continuando o consumo da fábrica de $100 \mathrm{t} / \mathrm{h}$, restariam 358,4 t/h de vapor para geração de energia. Sendo assim, nesta situação teria um excedente de vapor que seria desperdiçado e como dito anteriormente, a solução seria reduzir a alimentação para manter a geração de vapor em $240 \mathrm{t} / \mathrm{h}$.

A usina Sinimbu conta com uma área disponível para plantio da cana-de-açúcar de 26.104 hectares, divididos em 13.000 hectares próprios e 13.104 hectares pertencentes aos fornecedores da usina.

Utilizando os dados de produtividade média de cana-de-açúcar, quantidade de resíduos por hectare, foram calculados as quantidades de resíduos gerados na área dos canaviais da usina (Tabela 5).

Porém, como dito anteriormente, para poder ser utilizada como combustível as palhas e pontas não devem passar por processo de queimada. E como também já citado anteriormente, a média de mecanização no estado de Alagoas é de 50\%, portanto para o levantamento, utilizou-se apenas a área própria da usina, sendo assim uma boa estimativa. Com o resíduo já na indústria ele terá que passar por um tratamento para poder ser utilizado nas caldeiras. Ele poderá ser moído junto com a cana-de-açúcar no processo de moagem, o que não seria muito interessante, pois aumentaria o desgaste dos equipamentos. 
Tabela 4: Comparativo de geração de vapor entre bagaço e palhas e pontas.

\begin{tabular}{ccccc}
\hline Resíduo & $\begin{array}{c}\text { Alimentação } \\
(\mathrm{t} / \mathrm{h})\end{array}$ & $\begin{array}{c}\text { Energia Total } \\
(\mathrm{KJ} / \mathrm{h})\end{array}$ & $\begin{array}{c}\text { Vazão } \\
\text { Vapor Real }\end{array}$ & $\begin{array}{c}\text { Teórico } \\
(\mathrm{t} / \mathrm{h})\end{array}$ \\
\hline Bagaço & 40 & $(\mathrm{t} / \mathrm{h})$ & 80 & 104,18 \\
Palhas e & 40 & 275.395 .600 & 152,8 & 199 \\
pontas & & 526.079 .680 & & \\
\hline
\end{tabular}

Tabela 5: Quantidade de resíduo gerado em área da usina Sinimbu.

\begin{tabular}{cccc}
\hline \multirow{2}{*}{ Resíduo } & $\begin{array}{c}\text { Quantidade de Resíduo em } \\
\text { Área Própria } \\
(\mathrm{t})\end{array}$ & $\begin{array}{c}\text { Quantidade de Resíduo } \\
\text { em Área de }\end{array}$ & $\begin{array}{c}\text { Quantidade de } \\
\text { Resíduo na Área }\end{array}$ \\
& & Fornecedores (t) & Total \\
Bagaço & 189.150 & & 379.813 \\
Palhas e Pontas & 151.320 & 190.663 & 303.850 \\
\hline
\end{tabular}

O mais coerente seria a instalação de um equipamento para reduzir o tamanho das partículas deste resíduo e assim aumentando a superfície de contato.

$\mathrm{O}$ uso de um picador de palha atingiu resultados satisfatórios. O picador é um equipamento simples, composto por um rotor com facas fazendo com que a palha passe por um cesto, assim transformando as palhas de fibras longas em palha picada, ideal para a queima em caldeiras (FIGUEIREDO; NETO, 2012).

Após picada, as palhas e pontas podem ser alimentadas nas caldeiras junto com o bagaço. Porém, se quando se analisa somente as palhas e pontas, utilizando os resíduos disponíveis sabe-se que o potencial de geração é de 458,4 t/h de vapor alimentado $120 \mathrm{t} / \mathrm{h}$ de palha.

Como dito anteriormente, para manter a atual necessidade da fábrica era preciso alimentar apenas $62,82 \mathrm{t} / \mathrm{h}$. com a quantidade de resíduos disponível, pode gerar energia por aproximadamente 102 dias.

Tendo em vista que a geração de energia com os equipamentos atuais é de $18 \mathrm{MW} / \mathrm{h}$, que destes, apenas $6 \mathrm{MW} / \mathrm{h}$ são destinados a venda de energia elétrica, estimou-se a quantidade de energia que pode ser gerada nesses 113 dias, que foi de aproximadamente 15 mil MW. 
Segundo NASCIMENTO (2014), no mercado livre o valor do $\mathrm{MW} / \mathrm{h}$ esta atingindo valores superiores a $\mathrm{R} \$ 500,00$. Considerando o valor de $\mathrm{R} \$ 500,00$, a usina teria uma arrecadação com a venda deste excedente de energia elétrica na faixa de aproximadamente 7 milhões e 500 mil reais. Isto apenas utilizando as palhas e pontas, porém ainda se conta com a energia oriunda do bagaço, o que não está sendo calculado aqui, pois já é uma receita da usina.

\section{Conclusão}

Os resíduos de biomassa apresentam alto potencial energético, constituindo assim em alternativa econômica para o setor frente ao cenário de crise econômica verificado em grande parte das unidades do setor sucroenergético.

\section{REFERÊNCIAS}

COMPANHIA NACIONAL DE

ABASTECIMENTO (CONAB).

Acompanhamento da Safra Brasileira de

Cana-de-açúcar. Brasília: CONAB, 2013.

v.1. p. 1-14, 2013 Disponível em: <

http://www.conab.gov.br/OlalaCMS/uploads/a

rquivos/14_04_10_09_00_57_boletim_cana_p ortugues_-_4o_lev_-_13.pdf $>$. Acesso em: 23 de maio de 2014.

FIGUEIRÊDO, R. L. M. C.; NETO, J. M. A. Estudo da Viabilidade do Aproveitamento da Palha da Cana-de-Açúcar para

Cogeração de Energia no Setor

Sucroalcooleiro. Trabalho de conclusão de curso, Engenharia Elétrica, CESMAC, 2012.

NASCIMENTO, D. Bioeletricidade às escuras. RPA Cana como Negócio. Revista RPA News, Ano 12, ed. 159, p. 6, abr, 2014.
RANADE, P.; KAWRE, J. P. A seminar report on hydrogen from biomass, 2010. Disponível em:

<http://www.scribd.com/doc/7365674/hydroge n-from-biomass >.Acesso em: 10 abril. 2015.

SOUZA, E. A. de. Avaliação das Diversas Fontes e Tipos de Biomassa do Estado de Alagoas: Estudo de suas Características Físico-Químicas e de seu Potencial Energético. Maceió: UFAL, 2011. Tese de Doutorado em Ciências - Programa de Pós Graduação em Química e Biotecnologia, Instituto de Química e Biotecnologia, Universidade Federal de Alagoas, Maceió, 2011. 